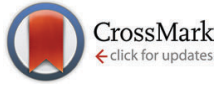

Cite this: Chem. Commun., 2014 50, 15623

Received 28th September 2014 Accepted 24th October 2014

DOI: $10.1039 / c 4 c c 07663$

www.rsc.org/chemcomm

\section{Identification of charge separated states in thymine single strands $\uparrow$}

\author{
Bert M. Pilles, ${ }^{a}$ Dominik B. Bucher, ${ }^{a}$ Lizhe Liu, ${ }^{a}$ Peter Gilch, ${ }^{b}$ Wolfgang Zinth ${ }^{\star a}$ and \\ Wolfgang J. Schreier*a
}

UV excitation of the DNA single strand (dT) $)_{18}$ leads to electronically excited states that are potential gateways to DNA photolesions. Using time-resolved infrared spectroscopy we characterized a species with a lifetime of $\sim 100$ ps and identified it as a charge separated excited state between two thymine bases.

The formation and decay of electronically excited states in DNA has been of major interest since the pioneering work on DNA photochemistry in the $1960 \mathrm{~s}^{1}{ }^{1}$ Based on the finding that the integrity of DNA is endangered by the mutagenic potential of UV induced photolesions, ${ }^{2,3}$ attempts to unravel the relevant damage pathways are directly bound to monitor the initial steps after UV absorption. Recent advances in time resolved techniques made it possible to follow DNA excitations in real time. ${ }^{4,5}$ It could be shown that single bases and mononucleotides predominantly decay to the electronic ground state via ultrafast internal conversion. In base sequences and double stranded DNA increasing contributions of longer lived excited states were detected. ${ }^{5}$ Thereby, excitons, exciplexes and excimer states with charge transfer character have been proposed to be responsible for prolonged excited state lifetimes. ${ }^{6-9}$

The most abundant UV induced DNA lesions occur between thymine bases, and all-thymine single strands became the focus of a series of time resolved experimental and computational studies. $^{10-17}$ Thereby, charge transfer (CT) states have been suggested to act as precursor species for the formation of (6-4) photoproducts in thymine sequences. ${ }^{17}$ Yet, until now charge transfer excited states between two thymine bases have not been

\footnotetext{
${ }^{a}$ Lehrstuhl für BioMolekulare Optik, Fakultät für Physik and Munich Center for Integrated Protein Science CIPSM, Ludwig-Maximilians-Universität München, Oettingenstr.67,80538 München, Germany.E-mail:wolfgang.schreier@gmx.net, wolfgang.zinth@physik.uni-muenchen.de

${ }^{b}$ Institut für Physikalische Chemie, Heinrich-Heine-Universität Düsseldorf, Universitätsstr. 1, 40225 Düsseldorf, Germany

$\dagger$ Electronic supplementary information (ESI) available: Materials and methods, Raw data and corrections, Identification of CT signature $\left(\mathrm{T}^{{ }^{+}}{ }_{-} \mathrm{T}^{*-}\right)$, Quantum yield of CT formation, Estimates on rate constants for CT and recombination. See DOI: $10.1039 / \mathrm{c} 4 \mathrm{cc} 07663 \mathrm{j}$
}

identified unequivocally. Moreover, earlier experiments in the UV and visible spectral range are not conclusive. Reaction dynamics on the 100 ps time scale have been observed in two independent studies on $(\mathrm{dT})_{18}$ and $(\mathrm{dT})_{20} \cdot{ }^{14,18}$ In one case the decay was tentatively assigned to a singlet $n \pi^{*}$ state. ${ }^{18}$ In the other case the decay was ascribed to the quenching of triplet states eventually leading to the formation of cyclobutane pyrimidine dimer (CPD) lesions. ${ }^{14}$ Neither of the studies reported direct evidence for the interpretations due to the lack of conclusive spectral signatures in the UV and visible spectral range monitored in these experiments.

In this study, we applied time resolved IR spectroscopy (TRIR) to reinvestigate the picosecond dynamics in the allthymine single strand $(\mathrm{dT})_{18}$. TRIR has been shown to allow the unequivocal spectral identification of electronically excited states, reaction intermediates and photoproducts..$^{8,13,19-21}$ The obtained data show that UV excitation in $(\mathrm{dT})_{18}$ leads to the formation of electronically excited states that decay to the electronic ground state on a 100 ps time scale. Comparison of the spectral signature with experimentally determined anionic and cationic thymine species provides unequivocal evidence that the observed species resemble charge separated states between two thymine bases. The latter are formed with a quantum yield of $\sim 0.07$.

Experimental data obtained for $(\mathrm{dT})_{18}$ in buffered $\mathrm{D}_{2} \mathrm{O}$ solution after excitation at $266 \mathrm{~nm}$ are given in Fig. 1. To emphasize the kinetics on the $100 \mathrm{ps}$ time scale the data have been corrected for absorption changes on the nanosecond time scale. In this procedure, experimental data between 400 ps and 600 ps were averaged and subtracted from the original data set (see ESI $\dagger$ ). The contour plot in Fig. 1 shows bleach signals (indicated in blue) at $1630 \mathrm{~cm}^{-1}, 1664 \mathrm{~cm}^{-1}$ and $1696 \mathrm{~cm}^{-1}$ matching the ground state absorption spectrum. Prominent positive absorption bands are located at $1570 \mathrm{~cm}^{-1}$ and $1760 \mathrm{~cm}^{-1}$ (indicated in red). The decay of the excited state absorption and the recovery of the ground state at $1664 \mathrm{~cm}^{-1}$ both occur on the $100 \mathrm{ps}$ time scale. Representative time traces at $1570 \mathrm{~cm}^{-1}$ (excited state absorption, red) and $1664 \mathrm{~cm}^{-1}$ (ground state bleach, blue) 


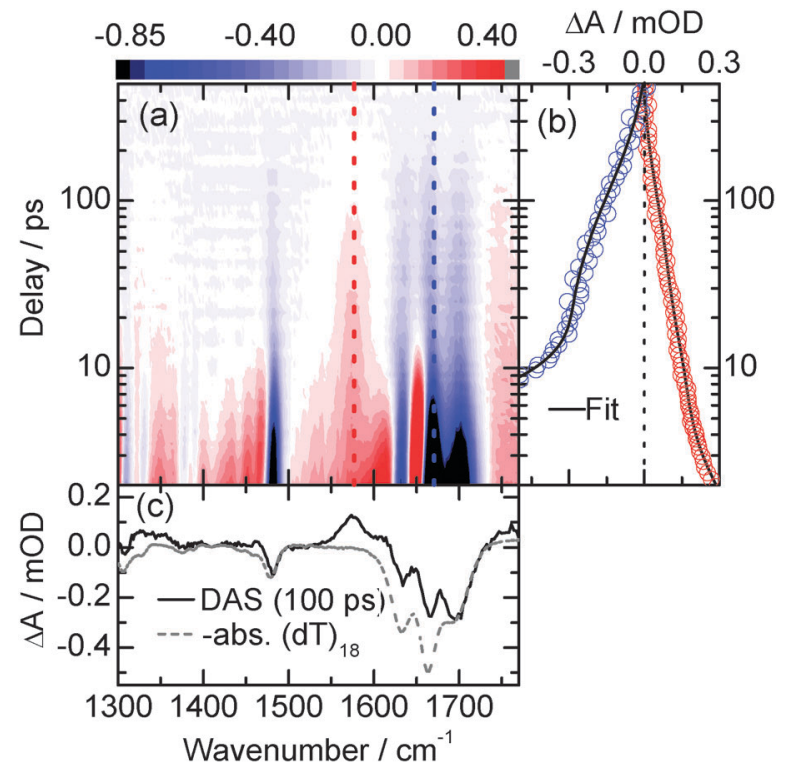

Fig. 1 Time resolved IR absorption changes of (dT) 18 after $266 \mathrm{~nm}$ excitation. (a) Contour representation of the transient absorption difference spectra corrected for the absorption changes at late delay times (see text for details). (b) Time dependence of the absorption changes (circles) recorded at $1570 \mathrm{~cm}^{-1}$ (red) and $1664 \mathrm{~cm}^{-1}$ (blue). Results of a global fitting procedure using a sum of two exponentials are given as solid lines. (c) The spectrum (DAS) associated with the 100 ps component obtained by this fit is given by the black solid line. The inverted ground state absorption spectrum of $(\mathrm{dT})_{18}$ is given as broken line for comparison.

underscore this assignment (Fig. 1b). The analysis of the data set relied on a global fitting routine. ${ }^{22}$ Thereby, a sum of exponentials convoluted with the experimental response served as trial function. The experimental data can be readily reproduced by a fast component representing signatures on the femtosecond to picosecond time scale including ultrafast internal conversion and cooling kinetics. ${ }^{13,23,24}$ Additionally, a slow decay component with a lifetime of $\sim 100 \mathrm{ps}$ is observed. The decay associated spectrum $\operatorname{DAS}(100 \mathrm{ps})$ of the latter is given in Fig. 1c together with the inverted ground state absorption of $(\mathrm{dT})_{18}$ (broken line) for comparison.

The nature of the species associated with the 100 ps decay component $\operatorname{DAS}(100 \mathrm{ps})$ will be discussed next. In a transient absorption study by Kwok et al. a species with a similar lifetime of $\sim 140$ ps was found for $(\mathrm{dT})_{20}$ in aqueous solution and interpreted as triplet decay. ${ }^{14}$ Yet, a recent study on $(\mathrm{dT})_{18}$ covering the nanosecond time scale excludes this interpretation. ${ }^{25}$ The latter study revealed that the lowest triplet state ${ }^{3} \mathrm{LT}$ of thymine exhibits a characteristic spectral signature with a triplet marker band around $1600 \mathrm{~cm}^{-1}$ that decays on the $10 \mathrm{~ns}$ time range. A comparison of the decay associated spectra of the triplet state with $\operatorname{DAS}(100 \mathrm{ps})$ is given in Fig. 2. Clearly, the signature of the triplet state (indicated by the red line) is missing in the DAS(100 ps). Based on the spectral signature and the different time scales an assignment to the lowest triplet state can be ruled out for the DAS(100 ps). Also the assignment to a triplet $n \pi^{*}$ state is unlikely.

The latter would be expected to serve as gateway to the lowest triplet state with $\pi \pi^{*}$ character. Yet, as can be seen from

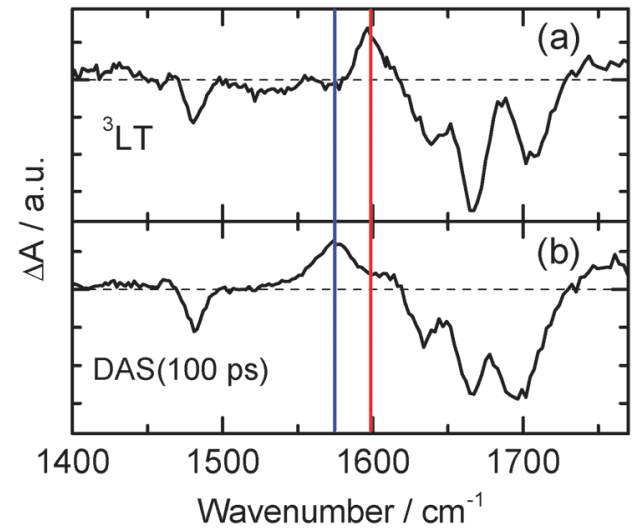

Fig. 2 Comparison of absorbance changes indicative of the decay of the lowest triplet state ${ }^{3}$ LT (a) and of the decay component DAS(100 ps) (b) obtained for $(\mathrm{dT})_{18}$. The position of the marker band for the lowest triplet state around $1600 \mathrm{~cm}^{-1}$ is indicated in red, the characteristic absorption of the 100 ps decay component around $1575 \mathrm{~cm}^{-1}$ is indicated in blue.

the data presented in Fig. 1 such a state is absent and the observed state related with the $\operatorname{DAS}(100 \mathrm{ps})$ decays directly to the starting material.

In an earlier study Kohler et al. were the first to observe an electronically excited species with a lifetime of $\sim 103$ ps in the all-thymine single strand $(\mathrm{dT})_{18 .}{ }^{18}$ The observed species was tentatively assigned to a singlet $n \pi^{*}$ state. ${ }^{18}$ Yet, the assignment to a singlet $n \pi^{*}$ state is unlikely in view of results obtained for TMP where a 100 ps decay component was not observed (see ESI $\dagger$ ). This suggests that the 100 ps component is not an intrinsic excited state of the thymine base, but is related to the interaction of thymine bases in the strand. It should be noted that this result contradicts the study by Kohler et al., where a $100 \mathrm{ps}$ time constant was also observed for TMP. ${ }^{18}$ Yet, preliminary experiments by the present authors on the transient dichroism of thymine and TMP have shown that such a 100 ps component of TMP can be assigned to orientational diffusion.

Based on the assumption that the excited state is associated with base interactions, excimer or exciplex states have to be taken into consideration. We propose that the observed species is due to an excimer state with significant charge transfer character, respectively a charge separated state on adjacent or separated thymine bases in the strand. To support our hypothesis we compare the observed DAS(100 ps) with the IR signatures of the corresponding cationic and anionic thymine species (for details see ESI $\dagger$ ). The cationic species $\mathrm{T}^{\circ+}$ was generated by twophoton ionization of $(\mathrm{dT})_{18}$ and its spectrum recorded by TRIR (Fig. 3a). Obtaining the anionic species $\mathrm{T}^{\bullet-}$ requires a reducing agent. Photo-excited adenine bases reduce the thymine moiety in TpA in agreement with recent results by Doorley et al. on the dinucleotide ApT. ${ }^{6}$ Therefore, a TRIR experiment on photo-excited TpA yields the decay spectrum of $\mathrm{T}^{\bullet-} \mathrm{pA}^{\bullet+}$ (see ESI, $\dagger$ Fig. S6a). In order to obtain the information on $\mathrm{T}^{\bullet-}$ the spectrum of $\mathrm{T}^{\bullet-} \mathrm{pA}^{\bullet+}$ must be corrected for the contribution of the cation radical $\mathrm{A}^{{ }^{+}}$ (see ESI, $\uparrow$ Fig. S6a). The latter cation signature was determined via two-photon ionization of $(\mathrm{dA})_{18}$ taking into account the specific 


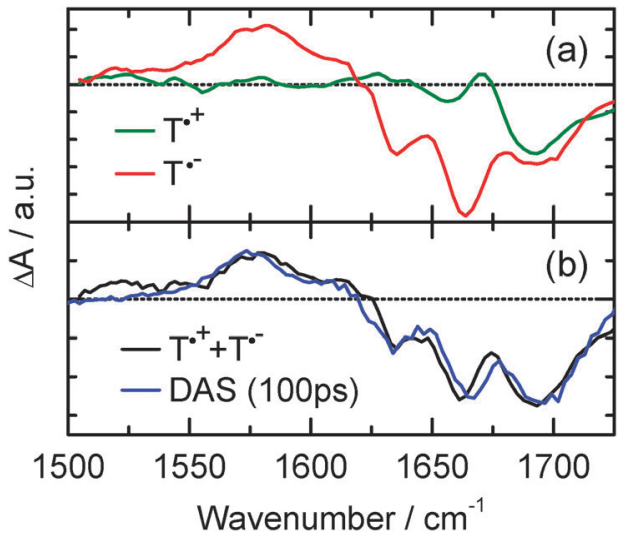

Fig. 3 (a) Signatures of the thymine cation $\left(\mathrm{T}^{\bullet+}\right)$ and anion $\left(\mathrm{T}^{\bullet-}\right)$ obtained for (dT) 18 in independent experiments. (b) Comparison between the charge transfer signature calculated from the cation and anion signals $\left(\mathrm{T}^{\bullet^{+}} \mathrm{pT}^{\boldsymbol{}^{-}-}\right)$ and DAS(100 ps).

spectral changes due to base stacking interactions in $(\mathrm{dA})_{18}$ (see ESI $\dagger$ ). The spectra of the cation $\mathrm{T}^{*+}$ and the anion $\mathrm{T}^{\bullet-}$ obtained by this procedure were added to yield a prediction for the spectrum of the CT state. A direct comparison of this spectrum and DAS(100 ps) is given in Fig. $3 \mathrm{~b}$. The excellent match between the signatures for CT state (black line) and $\operatorname{DAS}(100 \mathrm{ps})$ (blue line) shows that $\mathrm{DAS}(100 \mathrm{ps})$ is indeed a CT state and that CT states are present in all-thymine sequences after UV excitation. An estimate of the quantum yield for CT formation results in a value of $\varphi_{\mathrm{CT}} \sim 0.07$ (for details see ESI $\dagger$ ).

From the lifetime of the primary excitation in thymine derivatives $\left(\tau_{\mathrm{fl}} \approx 0.5-1\right.$ ps see ref. 26$)$ and the observed yield one can estimate that the rate constant for CT $k_{\mathrm{CT}} \approx \varphi_{\mathrm{CT}} / \tau_{\mathrm{fl}}$ is of the order of $10^{11} \mathrm{~s}^{-1}$. This value applies for a homogeneous situation where all excited thymines have the same probability for CT formation. For an inhomogeneous situation, i.e. when a geometry favourable for CT is realized only for a minority of thymines in the strand the reaction rate will be substantially higher. The charge recombination (CR) process occurs with a rate constant $k_{\mathrm{RC}}$ of $10^{10} \mathrm{~s}^{-1}$. The order of magnitude of these rate constants is consistent with predictions from electron transfer theory (see ESI $\dagger$ ). Electron transfer theory also suggests, that thymine in a triplet state - due to its lower energy would allow only a very slow CT (at a rate constant of $4 \times 10^{3} \mathrm{~s}^{-1}$ ). This matches our recent findings that the triplet excited thymine in $(\mathrm{dT})_{18}$ exhibits a lifetime of $10 \mathrm{~ns}$ and decays via the formation of a biradical and not via a CT state. ${ }^{25}$

In a recent quantum chemical study, CT states between thymine bases have been discussed to be the starting point for the formation of the (6-4) lesion. ${ }^{17}$ The decay of such a charge transfer state was predicted to yield oxetane intermediates, as precursors of the (6-4) adduct. Yet, as shown in Fig. 1a, we do not observe any other species formed with the decay of the CT states. Thus, the vast majority of the CT-states decays back to the electronic ground states of the starting material. However, since the quantum yield of (6-4) formation $\varphi_{6-4}$ is known to be very low (on the order of 0.005 ), ${ }^{7}$ a similar small yield for formation of the proposed oxetane intermediate might hamper its detection. The yield $\varphi_{6-4}$ and the yield of the CT state $\varphi_{\mathrm{CT}}$ reported here imply that less than a fraction of 0.1 of the CT population transforms into (6-4) photolesions - provided that the CT state is indeed the precursor.

In conclusion, time resolved IR spectroscopy was used to investigate the 100 picosecond dynamics of the all-thymine single strand $(\mathrm{dT})_{18}$ after UV excitation. We observed the formation of an electronically excited species that decays with a time constant of $\sim 100$ ps. By comparison with the experimentally determined signatures of cationic and anionic species of thymine compounds the latter could unequivocally be assigned to a charge transfer (CT) state. While CT states have been demonstrated for mixed DNA sequences before, the present study yields the first direct experimental assignment of a CT state between two thymine bases on the $100 \mathrm{ps}$ time scale. The latter is of major importance as CT states have been proposed to act as precursors for the formation of DNA photolesions. Yet, although the CT states are initially formed with a comparably high quantum yield of $\sim 0.07$, we did not see any indication for the formation of reaction intermediates or photolesions. Instead, the CT states decay predominantly to the electronic ground state.

This work was supported by the Deutsche Forschungsgemeinschaft (SFB 749, A5) and by the DFG-Clusters of Excellence 'Munich-Center for Advanced Photonics' and 'Center for Integrated Protein Science Munich' (CIPSM). P.G. acknowledges support by the Deutsche Forschungsgemeinschaft (Gi 349/3-2, Heisenberg professorship). We are grateful to Prof. Bern Kohler for fruitful discussions.

\section{Notes and references}

1 R. Beukers, A. P. M. Eker and P. H. M. Lohman, DNA Repair, 2008, 7, 530-543.

2 M. G. Friedel, M. K. Cichon and T. Carell, in CRC Handbook of Organic Photochemistry and Photobiology, ed. W. Horspool and F. Lenci, CRC Press LLC, 2004, pp. 141/141-141/122.

3 J. S. Taylor, Acc. Chem. Res., 1994, 27, 76-82.

4 C. E. Crespo-Hernandez, B. Cohen, P. M. Hare and B. Kohler, Chem. Rev., 2004, 104, 1977-2020.

5 C. T. Middleton, K. de La Harpe, C. Su, Y. K. Law, C. E. CrespoHernandez and B. Kohler, Annu. Rev. Phys. Chem., 2009, 60, 217-239.

6 G. W. Doorley, M. Wojdyla, G. W. Watson, M. Towrie, A. W. Parker, J. M. Kelly and S. J. Quinn, J. Phys. Chem. Lett., 2013, 4, 2739-2744.

7 A. Banyasz, T. Douki, R. Improta, T. Gustavsson, D. Onidas, I. Vaya, M. Perron and D. Markovitsi, J. Am. Chem. Soc., 2012, 134, 14834-14845.

8 D. B. Bucher, B. M. Pilles, T. Carell and W. Zinth, Proc. Natl. Acad. Sci. U. S. A., 2014, 111, 4369-4374.

9 T. Takaya, C. Su, K. de La Harpe, C. E. Crespo-Hernandez and B. Kohler, Proc. Natl. Acad. Sci. U. S. A., 2008, 105, 10285-10290.

10 A. Banyasz, I. Vaya, P. Changenet-Barret, T. Gustavsson, T. Douki and D. Markovitsi, J. Am. Chem. Soc., 2011, 133, 5163-5165.

11 A. T. Johnson and O. Wiest, J. Phys. Chem. B, 2007, 111, 14398-14404.

12 Y. K. Law, J. Azadi, C. E. Crespo-Hernandez, E. Olmon and B. Kohler, Biophys. J., 2008, 94, 3590-3600.

13 W. J. Schreier, T. E. Schrader, F. O. Koller, P. Gilch, C. E. CrespoHernandez, V. N. Swaminathan, T. Carell, W. Zinth and B. Kohler, Science, 2007, 315, 625-629.

14 W. M. Kwok, C. Ma and D. L. Phillips, J. Am. Chem. Soc., 2008, 130, 5131-5139. 
15 S. Marguet and D. Markovitsi, J. Am. Chem. Soc., 2005, 127, 5780-5781. 16 W. J. Schreier, J. Kubon, N. Regner, K. Haiser, T. E. Schrader, W. Zinth, P. Clivio and P. Gilch, J. Am. Chem. Soc., 2009, 131, 5038-5039.

17 R. Improta, J. Phys. Chem. B, 2012, 116, 14261-14274.

18 C. E. Crespo-Hernandez, B. Cohen and B. Kohler, Nature, 2005, 436, 1141-1144.

19 S. Laimgruber, W. J. Schreier, T. Schrader, F. Koller, W. Zinth and P. Gilch, Angew. Chem., Int. Ed., 2005, 44, 7901-7904.

20 M. K. Kuimova, A. J. Cowan, P. Matousek, A. W. Parker, X. Z. Sun, M. Towrie and M. W. George, Proc. Natl. Acad. Sci. U. S. A., 2006, 103, 2150-2153.
21 K. Haiser, B. P. Fingerhut, K. Heil, A. Glas, T. T. Herzog, B. M. Pilles, W. J. Schreier, W. Zinth, R. de Vivie-Riedle and T. Carell, Angew. Chem., Int. Ed., 2012, 51, 408-411.

22 H. Satzger and W. Zinth, Chem. Phys., 2003, 295, 287-295.

23 B. Kohler, J. Phys. Chem. Lett., 2010, 1, 2047-2053.

24 M. K. Kuimova, J. Dyer, M. W. George, D. C. Grills, J. M. Kelly, P. Matousek, A. W. Parker, X. Z. Sun, M. Towrie and A. M. Whelan, Chem. Commun., 2005, 1182-1184.

25 B. M. Pilles, D. B. Bucher, L. Liu, P. Clivio, P. Gilch, W. Zinth and W. J. Schreier, J. Phys. Chem. Lett., 2014, 5, 1616-1622.

26 T. Gustavsson, A. Sharonov and D. Markovitsi, Chem. Phys. Lett., 2002, 351, 195-200. 